\title{
The selfish pursuit of sex
}

SIR - No satisfactory explanation for the has been proposed that is based solely on the selective advantage (to organisms) derived from genetic shuffling, a primary consequence of meiosis ${ }^{1}$. Several theories do see genetic shuffling as a byproduct of some other selective advantage, and one, 'the molecular symbiont' hypothesis, would have it as a primary advantage, but an advantage accruing to genetic parasites, not organisms ${ }^{1}$. This idea, first proposed by Hickey ${ }^{2}$ and by Rose ${ }^{3}$, has the weakness that no gene that is an integral origin and evolution of sex in eukaryotes

found in archaebacterial DNA polymerase genes, indicates that $\mathrm{HO}$ endonuclease probably arose from a homing protein-intron at roughly the same time that Saccharomyces diverged from Candida (see figure), about 135 million years ago ${ }^{7}$. The alternative, that protein-introns are degenerate derivatives of $\mathrm{HO}$ endonuclease $^{5}$, is a far less parsimonious interpretation of the phylogeny.

Although there are cases of selfish elements directing the fusion of mitochondria to promote their own spread in eukaryotes $^{8}$, the relationship between HO endonuclease and protein-introns is the first strong evidence for a mobile element actually becoming integrated into the sexual cycle of the nucleus. To understand the significance of this relationship, one must appreciate the effect that frequent mating-type switching could have on the spread of a transposon. In a heterothallic population under certain conditions, such as a discontinuous or patchy population distribution with little mixing, subpopulations of essentially clonal lineages of one mating type may arise. Haploid individuals from a population bearing an element that increases the frequency of mating-type switching would more often find compatible mates under these conditions: within their original clonal lineage they endonuclease, fungal V-ATPase protein introns and archaebacterial DNA polymerase protein-introns. The upper tree was obtained by parsimony analysis (using PAUP 3.1 program), whereas the lower tree was generated by neighbour-joining of a protein-distance matrix (using PHYLIP 3.5 programs) and protein maximum likelihood (using MOLPHY 2.2 programs), inferred from 57 clearly alignable amino-acid positions. The exact topology of the fungal sequences cannot be discerned, but all methods confirm with strong statistical support that $\mathrm{HO}$ and the two ATPase protein-introns are most closely related and diverged at about the same time.

part of the eukaryotic sexual process has been shown to have been derived from a selfish mobile element ${ }^{1,4}$. In fact, a good example has been in the literature for some time, but its significance has never been recognized.

Recently, a new class of selfish mobile element has been discovered, the protein-intron (intein). These elements are endonucleases that are also known to 'home', a particular type of mobility that uses double-stranded-break repair to invade uninfected alleles 5 . Interestingly, these mobile elements are also related to the endonuclease encoded by the homothallic switching (HO) gene in the yeast Saccharomyces cerevisiae, the factor responsible for initiating a mating-type switching cascade in this organism ${ }^{6}$. Closer examination reveals that $\mathrm{HO}$ is most closely related to the V-type ATPase proteinintrons found in both Saccharomyces and the related Candida tropicalis. Phylogenetic analysis of these sequences, together with their next closest relatives, protein-introns mating-type switching was to favour diploidy, a measure that improved the organism's ability to repair damaged $\mathrm{DNA}^{6}$. We suggest, however, that by assuming this role in mating-type switching, the mobile protein-intron may have improved its own chances of propagation. If this proposal is correct, the establishment of $\mathrm{HO}$ in the ancestral Saccharomyces population was not primarily the consequence of organism-level selection for more frequent mating-type switching ${ }^{6}$, but rather may have been the result of a selfish transposon which had cleverly exploited a pre-existing mating-type switching process to speed its own spread.

Patrick J. Keeling, Andrew J. Roger

Department of Biochemistry,

Dalhousie University, Halifax,

Nova Scotia B3H 4H7, Canada

1. Hickey, D. A. J. Hered. 84, 410-414 (1993).

2. Hickey, D. A. Genetics 101, 519-531 (1982).

3. Rose, M. R. J. theor. Biol. 101, 137-147 (1983).

4. Bell, G. J. Hered. 84, 351-359 (1993).

5. Gimble, F. S. \& Thorner, J. Nature 357, 301-306 (1992).

6. Herskowitz, I. Microbiol. Rev. 52, 536-553 (1988).

7. Berbee, M. L. \& Taylor, J. W. Can. J. Bot. 71, 1114-1127 (1993).

8. Hurst, L. D. Nature 354, 23-24 (1991).

\section{Neanderthal}

\section{computer skulls}

SIR - Fossil specimens can be restored, measured and replicated without physical contact using a combination of computerized tomography (CT), computer-assisted reconstruction and rapid prototyping. We have used this procedure to reconstruct and reinterpret fragments of a Neanderthal child's skull from Devil's Tower in Gibraltar (Fig. $1 a, b)$. We have developed an interactive software package to generate accurate object surfaces from serial CT data which permits three-dimensional morphometry and computer-assisted reconstruction of fossils. Rapid prototyping of computergenerated reconstructions was achieved using stereolithography (layer-by-layer production of successive thin profiles by laser-induced polymerization of a photosensitive liquid).

The Devil's Tower specimen is represented by five individual fragments: incomplete mandible, right maxilla, right temporal, fused frontal bones and left parietal. Of these original fragments, only the last two articulate directly. All fragments were originally attributed to a single individual ${ }^{1}$ with an estimated age at death of 3-4 years ${ }^{2,3}$; but it has also been suggested that the fragments came from two separate individuals of different ages ${ }^{4}$.

Using a graphics workstation equipped with a stereo monitor (Onyx, Silicon Graphics; Mountain View, California), three-dimensional skull reconstruction was achieved in four stages (Fig. 1a). (1) The 